\title{
Improvement of sensitivity to tamoxifen in estrogen receptor-positive and Herceptin-resistant breast cancer cells
}

\author{
Bin Chen ${ }^{1}$, Yuanzhong Wang ${ }^{1}$, Susan E Kane ${ }^{2}$ and Shiuan Chen ${ }^{1}$ \\ ${ }^{1}$ Department of Surgical Research and 2Division of Molecular Medicine, Beckman Research Institute of the City of Hope, Duarte, California 91010, USA \\ (Correspondence should be addressed to S Chen; Email: schen@coh.org)
}

\begin{abstract}
ERBB2 overexpression in estrogen receptor (ER)-positive breast cancer cells such as BT474 (BT) cells has been found to confer resistance to tamoxifen, and suppression of ERBB2 improves the antiproliferative effects of tamoxifen. In this study, the responsiveness to tamoxifen in the BT/HerR, Herceptin-resistant BT cell lines established through constant Herceptin exposure, was evaluated. Compared with BT cells, improvement of sensitivity to tamoxifen in BT/HerR was demonstrated by ER functional analysis and cell proliferation assay. Tamoxifen in the resistant cell line was found to inhibit 17 $\beta$-estradiolstimulating estrogen-responsive gene $p S 2$ expression more effectively than in BT cells in real-time PCR assay. Western blot analysis showed that cross-phosphorylation between ER and downstream components of ERBB2 was attenuated in BT/HerR cells. ER redistribution from cytoplasm to nucleus could be found in these cells through immunofluorescence and confocal studies, and importantly, chromatin immunoprecipitation studies demonstrated that tamoxifen induced occupancy of the pS2 promoter by ER and nuclear receptor corepressor (NCOR1) instead of coactivator NCOA3 in these cells. Finally, combination of tamoxifen and Herceptin was found to improve the sensitivity of BT/HerR cells to Herceptin. Our results suggest that the ER genomic pathway in the ER-positive and Herceptin-resistant breast cancer cells may be reactivated, allowing tamoxifen therapy to be effective again, and a combination of tamoxifen and Herceptin can be a potential therapeutic strategy for ER-positive and Herceptin-resistant human breast cancer.
\end{abstract}

Journal of Molecular Endocrinology (2008) 41, 367-377

\section{Introduction}

The ERBB2 oncogene encodes a $185 \mathrm{kDa}$ transmembrane tyrosine kinase receptor, belonging to the epidermal growth factor receptor family that is amplified or overexpressed in $30 \%$ of breast cancers and is a poor prognostic factor (Slamon et al. 1989). Trastuzumab/ Herceptin is a humanized monoclonal antibody directed against ERBB2, which inhibits growth and proliferation of cancer cells overexpressing ERBB2 (Carter et al. 1992). Herceptin has also shown efficacy in combination with conventional chemotherapeutic agents (Dieras et al. 2001). However, while ERBB2-overexpressing cell lines are shown to be sensitive to Herceptin, greater than $70 \%$ of patients whose tumors overexpress ERBB2 do not respond to Herceptin monotherapy (Vogel et al. 2002). In addition, a significant percentage of women who initially respond to Herceptin may develop acquired resistance to therapy after prolonged treatment. The precise mechanism of Herceptin resistance has not been fully characterized. Overexpression of constitutively active Akt (Clark et al. 2002), IGF receptor (Lu et al. 2001), loss of PTEN function (Nahta et al. 2006), or MUC4/sialomucin complex (Price-Schiavi et al. 2002) may potentially be involved in the resistance phenotype in breast cancer. Many strategies of combination therapy for Herceptin-resistant breast cancer cells have been considered, i.e. the combination of
Herceptin with EGFR kinase inhibitors such as AG1478 or Iressa (Lenferink et al. 2000, Moasser et al. 2001), and a COX2-inactive PDK1 inhibitor, OSU-03012 (Tseng et al. 2006). New potential therapeutic strategies, such as the combination of Herceptin with IGF1R kinase inhibitors or anti-IGF1R antibodies, are being evaluated.

More than $60 \%$ of human breast cancers are estrogen receptor (ER)-positive. Until now, the selective ER modulator (SERM), tamoxifen, is the most common endocrine therapy used for these cancers. While $\sim 50$ $60 \%$ of ER-positive breast cancer patients benefit from tamoxifen, some of the ER-positive breast cancer patients who do not respond to tamoxifen have overexpression of ERBB2 (Slamon et al. 1989). There has been much experimental evidence to suggest that tamoxifen resistance in breast cancer cells involves overexpression of ERBB2 and its crosstalk with ER, which is associated with ER nongenomic activity (Pietras et al. 1995, Shou et al. 2004). Tamoxifen or estrogen (17 $\beta$-estradiol, $\mathrm{E}_{2}$ )-bound membrane/cytoplasmic ER, through multiple interactions with signaling intermediate molecules such as SH2 (Song et al. 2002) and modulator of nongenomic action of ER (Wong et al. 2002), can activate the EGFR/ ERBB2 signaling pathway. Activated downstream kinases, e.g., phosphatidylinositol 3'-kinase (PI3K)/Akt and the MAPK3/1/MAPK, can phosphorylate nuclear ER and its coactivators (Kato et al. 1995, Sun et al. 2001, Schiff et al.

DOI: 10.1677/JME-08-0026 Online version via http://www.endocrinology-journals.org 
2004, Shou et al. 2004), thus up-regulating genomic ER activity and enhancing gene expression including genes in the EGFR/ERBB2 pathways, which in turn further augment EGFR/ERBB2 signaling, thus completing the cooperative cycle between the two activities of ER and their crosstalk with the EGFR/ERBB2 signaling pathway. In ERBB2-overexpressing cells, the resulting activation of downstream kinases can lead to tamoxifen resistance by modifying the activity of various transcription factors and blocking the inhibitory effects of tamoxifen on nuclear ER. Corresponding to this observation, a variety of in vitro and in vivo models have shown that suppression of ERBB2 improves the antiproliferative effects of tamoxifen (Pietras et al. 1995, Tang et al. 1996, Kurokawa et al. 2000), again demonstrating a causal association between ERBB2 overexpression and acquisition of resistance to endocrine therapy such as tamoxifen.

To explore the mechanism of Herceptin resistance and find the potential therapeutic strategy for Herceptinresistant human breast carcinomas, several clones of BT474 (BT) human breast carcinoma cells that are resistant to Herceptin (BT/HerR) were isolated and identified over a 5-month selection process (Chan et al. 2005). BT is an ER-positive invasive human breast ductal carcinoma cell line with very high ERBB2 expression, which is very sensitive to Herceptin but resistant to tamoxifen, and has been used extensively for investigating the mechanisms of Herceptin and ERBB2 biology (Yakes et al. 2002, Zhou et al. 2007). BT/HerR subclones appear to have acquired a Herceptin-resistant PI3K signaling mechanism, because the downstream PI3K/ Akt signaling pathway is sustained in BT/HerR cells in the presence of Herceptin, but significantly down-regulated in BT cells exposed to Herceptin (Chan et al. 2005). Our previous results showed that, while total and phosphorylated cellular ERBB2 protein remained high, phosphorylated Akt levels in the presence of Herceptin in BT/HerR subclones were decreased compared with wild-type BT cells in the absence of Herceptin (Chan et al. 2005). Based on these findings and the known crosstalk between ER and ERBB2 in tamoxifen resistance, we hypothesized that $\mathrm{BT} /$ HerR and BT cells might have different responses to $\mathrm{E}_{2}$ and endocrine treatment, such as tamoxifen. This study was undertaken to explore the sensitivity to tamoxifen in the BT/HerR cells, and our results suggest that combined therapy with Herceptin and tamoxifen might be a viable therapeutic strategy for ER-positive and Herceptin-resistant human breast carcinomas.

\section{Materials and methods}

\section{Cell culture and reagents}

BT cells were routinely cultured in DMEM with $4.0 \mathrm{mM}$ L-glutamine, $4500 \mathrm{mg} / 1$ glucose (Hyclone, Logan, UT,
USA) supplemented with $10 \%$ heat-inactivated fetal bovine serum, $1 \%$ penicillin/streptomycin, and $1 \mathrm{mM}$ sodium pyruvate (Gibco BRL). BT/HerR0.2 clone D and $\mathrm{BT} /$ HerR1.0 clone $\mathrm{E}$ were maintained in the same medium with the continuous presence of 0.2 and $1 \mu \mathrm{mol} / 1$ Herceptin respectively (Chan et al. 2005). $\mathrm{E}_{2}$-driven cell assays were performed with steroid-deprived medium, where DMEM and FBS were replaced with DMEM without phenol red and charcoal-stripped FBS (Hyclone). Herceptin (Genentech, South San Francisco, CA, USA) was purchased from the City of Hope National Medical Center Pharmacy (Duarte, CA, USA), $\mathrm{E}_{2}$, and tamoxifen from Sigma Chemicals and the pure antiestrogen ICI 182780 (ICI) from Tocris (Ellisville, MO, USA).

\section{CellTiter 96 AQueous proliferation assay}

The response of $\mathrm{BT}$ or $\mathrm{BT} / \mathrm{HerR}$ cells to tamoxifen or Herceptin alone, or a combination of both agents, was examined using CellTiter 96 AQueous cell proliferation assay (Promega) according to the manufacturer's protocol. The cells were steroid deprived for $72 \mathrm{~h}$ and seeded in 96-well plates. After overnight incubation, steroiddeprived media containing $1 \mu \mathrm{M} \mathrm{E}_{2}, 1 \mu \mathrm{M}$ tamoxifen or indicated concentrations of Herceptin, or combination of both agents were added to the cells and left for 6 days on the cells. Each data point was performed in triplicate. At the indicated times, cells were incubated in the presence of $20 \mu \mathrm{l}$ CellTiter 96 AQueous One Reagent per $100 \mu \mathrm{l}$ medium for $1 \mathrm{~h}$, and the reaction was measured colorimetrically at $490 \mathrm{~nm}$ in a SpectraMax M5 Reader (Molecular Devices, Sunnyvale, CA, USA). Background levels were determined in medium supplemented with DMSO at the appropriate dose, and findings were recorded as mean \pm s.D.

\section{Real time RT-PCR analysis}

The cells were deprived of steroid for $72 \mathrm{~h}$ and incubated in the presence or absence of $1 \mathrm{nM} \mathrm{E} \mathrm{E}_{2}$ with or without $1 \mu \mathrm{M}$ tamoxifen for $24 \mathrm{~h}$. Whole-cell RNA was prepared using TRIzol Reagent (Invitrogen) and first-strand cDNA was synthesized using SuperScript III Reverse Transcriptase (Invitrogen), according to the manufacturer's instructions. Real-time PCR was performed using the iQ5 Real-Time PCR Detection System (Bio-Rad). The reactions were carried out in a 96-well plate using SYBR Green Supermix (Bio-Rad) according to the manufacturer's instructions. Primers used were as follows: pS2, 5'-ATACCATCGACGTCCCTCCA-3' (forward), primer $5^{\prime}$-CACCTCAGACACGCTT-3' (reverse); $\beta$-actin, $5^{\prime}$-AGAAGGAGATCACTGCCCTGGCACC-3' (forward), $5^{\prime}$-CCTGCTTGCTGATCCACATCTGCTG-3' (reverse). PCRs were performed with HotStarTaq DNA polymerase (Qiagen) under the following conditions: a preheating 
at $94{ }^{\circ} \mathrm{C}$ for $15 \mathrm{~min}$, followed by 30 cycles at $94{ }^{\circ} \mathrm{C}$ for $30 \mathrm{~s}$, $60{ }^{\circ} \mathrm{C}$ for $30 \mathrm{~s}, 72^{\circ} \mathrm{C}$ for $1 \mathrm{~min}$, followed by a $72^{\circ} \mathrm{C}$ extension for $10 \mathrm{~min}$. Each sample was analyzed in triplicate, and $\beta$-actin was used as a control. Relative quantification of $p S 2$ gene expression was evaluated by utilizing the cycle threshold $\left(C_{\mathrm{t}}\right)$. The $C_{\mathrm{t}}$ values for each cell were subtracted from the respective $C_{\mathrm{t}}$ value of the $\beta$-actin control, resulting in the $\Delta C_{\mathrm{t}}$ value. The $\Delta C_{\mathrm{t}}$ value for DMSO-treated cells was used as a constant that was subtracted from all the other $\Delta C_{\mathrm{t}}$ values to determine $\Delta \Delta C_{\mathrm{t}}$ value. Fold changes were then generated for each treated cells by calculating $2^{-\Delta \Delta C_{\mathrm{t}}}$.

\section{Transient transfection and luciferase ER reporter assays}

BT or BT/HerR cells were transfected with pGL3 (ERE) ${ }_{3}$-luciferase vector using Lipofectamine 2000 (Invitrogen) according to the protocol of the manufacturer. A total of $1.5 \times 10^{5}$ cells per well were seeded onto 24-well plates and incubated overnight. A mixture of $1 \mu \mathrm{g}$ of the reporter plasmid, $1.5 \mu$ l Lipofectamine 2000 and OptiMEM (Invitrogen) was added into each well and after a 4-h incubation, cells were cultured with fresh medium containing DMSO or $1 \mathrm{nM} \mathrm{E}_{2}$ with or without $1 \mu \mathrm{M}$ tamoxifen or $10 \mathrm{nM}$ ICI in phenol redfree medium with $10 \%$ charcoal-dextran-treated FBS. After a 24-h incubation following the transfection, the cells were lysed in $1 \times$ Reporter Lysis Buffer (Promega). Luciferase activity was measured using the Luciferase Assay System (Promega). Protein concentration was measured by the method of Bradford (Bio-Rad; Bradford 1976). The relative luciferase activity was calculated by dividing the light unit of luciferase activity by protein concentration of each sample.

\section{Immunofluorescence and confocal studies}

The cells were cultured in regular media ( $10 \%$ serum) or steroid-deprived media in the absence or presence of $1 \mathrm{nM} \mathrm{E}$. Subcellular localization of ESR1 in BT or BT/HerR was determined by indirect immunofluorescence. Cells grown on glass cover slips were fixed in ice-cold methanol:acetone $(1: 1)$ at $4{ }^{\circ} \mathrm{C}$ for $10 \mathrm{~min}$. The cells were incubated with the primary antibodies, mouse ER (F-10) antibody (Santa Cruz Biotechnology, Santa Cruz, CA, USA) or ER [6F11] antibody (Abcam, Cambridge, MA, USA) at $4{ }^{\circ} \mathrm{C}$ for overnight. After being washed three times in PBS, cells were then incubated with FITC-labeled (green) secondary antibody (Chemicon, Temecula, CA, USA). The VECTASHIELD Mounting Medium with DAPI (Vector Laboratories, Burlingame, CA, USA) was used to co-stain the DNA and was color coded in blue. Confocal analysis was performed with a Zeiss laser-scanning confocal microscope (Carl Zeiss, Oberkochen, Germany). Each image represents Z-sections at the same cellular level and magnification.

\section{Cell extracts and western analysis}

The cells were deprived of steroid for $72 \mathrm{~h}$ and serum starved for $24 \mathrm{~h}$, and then treated with DMSO, $1 \mathrm{nM} \mathrm{E}_{2}$, or $1 \mu \mathrm{M}$ tamoxifen for $24 \mathrm{~h}$. Isolation of cell extracts and western blot analysis was done as previously described (Lou et al. 2007). The following antibodies were used: total ESR1 and ERBB2, phospho-Ser118ESR1, phospho-Ser167-ESR1, total and phospho-forms of Akt $\left(\operatorname{Ser}^{437}\right)$, and MAPK $\left(\mathrm{Thr}^{202} / \mathrm{Tyr}^{204}\right.$; Cell Signaling, Boston, MA, USA). $\beta$-actin (Chemicon) was used as a control for equal loading and transfer. Quantitative densitometry of the immunoimages was performed using the Model GS-700 Imaging Densitometer with Molecular Analyst Software (Bio-Rad), and expressed as the ratios to the density of $\beta$-actin bands. Phosphorylation levels of the experiments were estimated by densitometry, and were calculated as the ratio of $\mathrm{pMAPK} / \mathrm{tMAPK}$, pAkt/tAkt, or $\mathrm{pER} / \mathrm{tER}$.

\section{Chromatin immunoprecipitation (ChIP) assays}

The cells were deprived of steroid for $72 \mathrm{~h}$ and serum starved for $24 \mathrm{~h}$, and then treated with DMSO, $1 \mathrm{nM}$ $\mathrm{E}_{2}$, or $1 \mu \mathrm{M}$ tamoxifen (Tam) for $45 \mathrm{~min}$. ChIP assays were carried out with the ChIP assay kit (Upstate, Charlottesville, VA, USA) as previously described (Lou et al. 2007). One-tenth of the immunoprecipitated DNA and $1 \%$ of the input DNA were analyzed by PCR. Antibodies used for ChIP assays included: NCOA3 and NCOR1 (Affinity Bioreagents, Golden, CO, USA), ESR1 and histone deacetylase 3 (HDAC3; Santa Cruz). PCRs were performed with HotStarTaq DNA polymerase (Qiagen) under the following conditions: a preheating at $94{ }^{\circ} \mathrm{C}$ for $15 \mathrm{~min}$, followed by 35 cycles at $94^{\circ} \mathrm{C}$ for $30 \mathrm{~s}, 60{ }^{\circ} \mathrm{C}$ for $30 \mathrm{~s}, 72^{\circ} \mathrm{C}$ for $1 \mathrm{~min}$, followed by a $72^{\circ} \mathrm{C}$ extension for $10 \mathrm{~min}$. The PCR products were analyzed by electrophoresis in a $1.5 \%$ agarose gel, which was then stained with ethidium bromide and photographed under u.v. illumination. Primers for the promoter region of the $p S 2$ gene: $5^{\prime}$ TTCATGAGCTCCTTCCCTTC-3' (forward) and $5^{\prime}$ ATGGGAGTCTCCTCCAACCT-3' (reverse). Representative experiments from at least three independent experiments are shown.

\section{Statistical analysis}

Overall differences between control and treatment groups, or differences between different treatment groups, were determined by two-way ANOVA. Direct 
comparisons between control and treatment effects were assessed using a Student's $t$-test. Differences were considered significant at $P<0 \cdot 05$.

\section{Results}

\section{Different sensitivity of tamoxifen in BT and BT/HerR cells}

To determine whether BT/HerR and BT cells might have different sensitivities to $\mathrm{E}_{2}$ and endocrine treatment, we first investigated the response of BT and $\mathrm{BT} / \mathrm{HerR}$ cells to endocrine therapy. Current endocrine therapy of breast cancer includes SERMs such as tamoxifen, the pure ER antagonist ICI 182780 (fulvestrant), and aromatase inhibitors such as letrozole. Because very low levels of aromatase activity were found in the BT and BT/HerR cells (data not shown), only the response to tamoxifen or ICI was examined. Two kinds of BT/HerR cells (BT/HerR0.2 and BT/HerR1.0 respectively) were selected to estimate the different sensitivity of BT cells to tamoxifen or ICI. In the ER functional analysis, while both the BT and $\mathrm{BT} / \mathrm{HerR}$ cells were found to be responsive to $\mathrm{E}_{2}$, $\mathrm{BT} /$ HerR cells were more sensitive to tamoxifen than BT cells (Fig. 1A). In BT/HerR0.2 or BT/HerR1.0 cells, tamoxifen could significantly decrease $\mathrm{E}_{2}$-stimulated
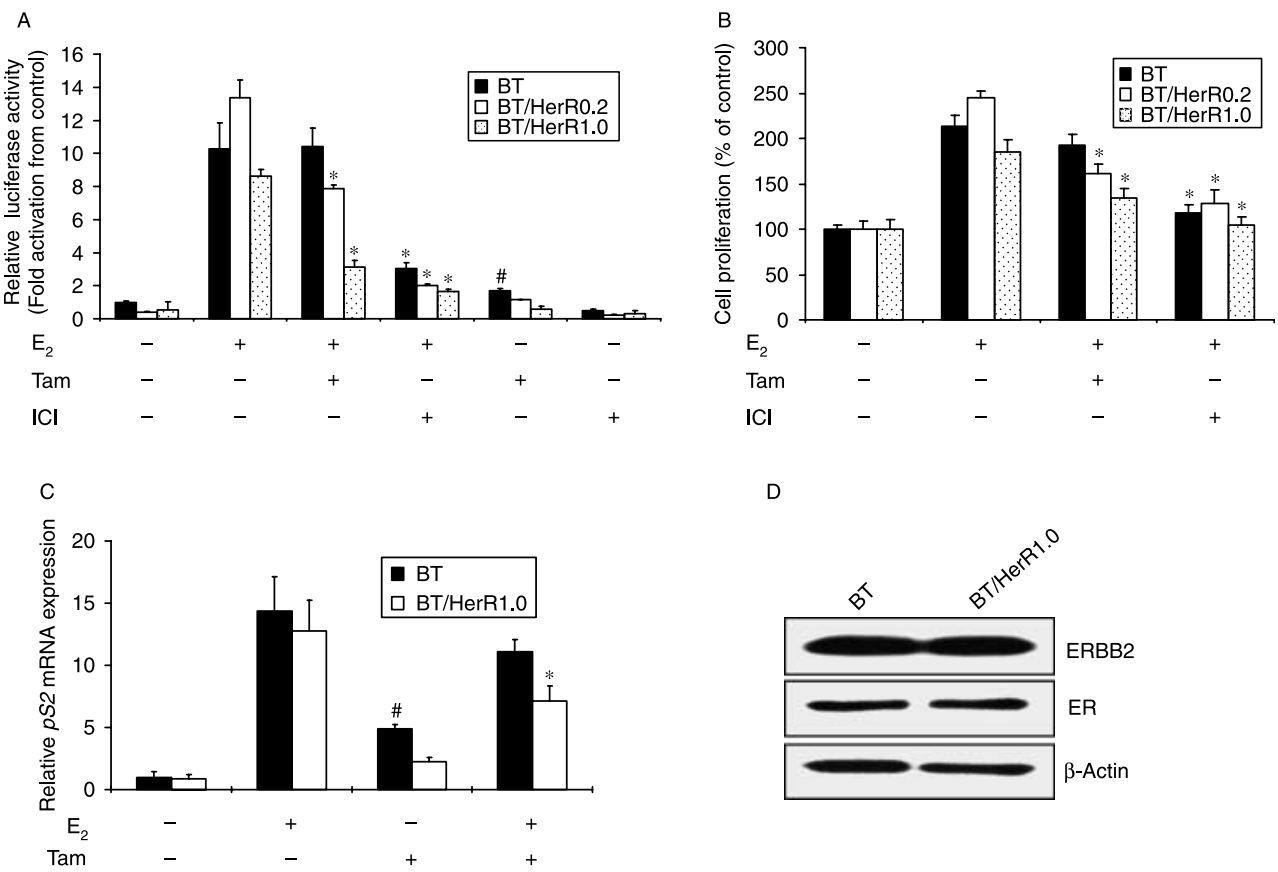

D

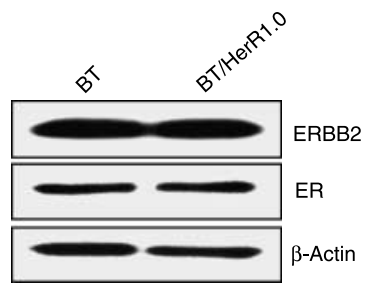

Figure 1 Different sensitivity to tamoxifen in the BT and BT/HerR cells. (A) Effect of tamoxifen and ICI on ER-mediated transcription in BT, BT/HerR0.2, and BT/HerR1.0 cells. The cells were transiently transfected with an ERE-containing luciferase reporter construct (ERE) in steroid-deprived medium, followed by a $16 \mathrm{~h}$ incubation in the presence or absence of $1 \mathrm{nM} \mathrm{E}_{2}$ with or without $1 \mu \mathrm{M}$ tamoxifen (Tam) or $10 \mathrm{nM} \mathrm{ICl}$. Normalized luciferase activity from triplicate wells was expressed relative to the luciferase activity of each cell line in the absence of $\mathrm{E}_{2}$, tamoxifen, and ICI. (B) Effects of tamoxifen and ICI on the proliferation in BT, $\mathrm{BT} / \mathrm{HerR} 0.2$, and BT/HerR1.0 cells. The cells were deprived of steroid for $72 \mathrm{~h}$ and then incubated in the presence or absence of $1 \mathrm{nM} \mathrm{E}_{2}$ with or without $1 \mu \mathrm{M}$ tamoxifen or $10 \mathrm{nM} \mathrm{ICl}$ for 6 days. Cell proliferation from triplicate wells was expressed relative to the optical density at $490 \mathrm{~nm}$ of each cell line in the absence of $E_{2}$, tamoxifen, and ICl. Results in $(A)$ and $(B)$ are expressed as mean \pm s.D. of at least three separate experiments. ${ }^{*} P<0.05$ compared with control in the presence of $E_{2}$. ${ }^{\#} P<0.05$ compared with control in the absence of $E_{2}$, tamoxifen, and ICI. (C) Real-time PCR analysis of the estrogen-responsive gene $p S 2$ in the BT and BT/HerR cells. BT and BT/HerR1.0 cells were deprived of steroid for $72 \mathrm{~h}$ and incubated in the presence or absence of $1 \mathrm{nM} \mathrm{E}_{2}$ with or without $1 \mu \mathrm{M}$ tamoxifen for $24 \mathrm{~h}$. The relative expression of $\mathrm{pS} 2$ in various treated cells was analyzed using $2^{-\Delta \Delta C_{\mathrm{t}}}$, which represents the expression of the $p S 2$ gene in various treated cells normalized to $\beta$-actin, relative to the normalized expression of pS2 in control cells. Results are expressed as mean \pm s.D. of at least three separate experiments. ${ }^{\star} P<0.05$ compared with control in the presence of $E_{2} . P<0.05$ compared with control in the absence of $E_{2}$ and tamoxifen. (D) Levels of ER and ERBB2 receptors in the BT and BT/HerR cells. BT and BT/HerR1.0 cells were cultured in regular media to $80 \%$ confluence. Lysates of cells were prepared and tested for ER and ERBB2 content by immunoblot analysis. $\beta$-actin was used as a control for equal loading and transfer. Blots are representative of three experiments. 
luciferase activity, confirming its antagonistic function. However, in BT cells, tamoxifen had no effect on $\mathrm{E}_{2}$-stimulated luciferase activity and, inversely, tamoxifen alone increased ERE-luciferase transcription to 1·7fold above basal level in BT cells, indicating a partial agonist function. In addition, the $\mathrm{BT}$ and $\mathrm{BT} / \mathrm{HerR}$ cells responded similarly to pure ER antagonist ICI, which could abrogate the estrogen signal in both types of cells by accelerating ESR1 degradation. To elucidate the biological significance of this difference in tamoxifen function in the BT and BT/HerR cells, we performed a cell proliferation assay and found that the BT and BT/HerR cells had the same response to ICI, but not to tamoxifen. BT cells were resistant to tamoxifen treatment, whereas $\mathrm{E}_{2}$-driven proliferation of $\mathrm{BT} /$ HerR cells was inhibited by tamoxifen $(57 \%$ inhibition in BT/HerR0.2 cells and $60 \%$ inhibition in BT/HerR1.0 cells; Fig. 1B). To further demonstrate the different sensitivities of tamoxifen in the BT and $\mathrm{BT} /$ HerR cells, levels of the estrogen-responsive gene $p S 2$ mRNA in these two kinds of cell lines were analyzed by real-time PCR. Tamoxifen was found to inhibit $\mathrm{E}_{2}$-stimulated $\mathrm{pS} 2$ expression in $\mathrm{BT} / \mathrm{HerR} 1.0$ cells more effectively than in BT cells (Fig. 1C). In addition, tamoxifen alone in the absence of $\mathrm{E}_{2}$ in $\mathrm{BT}$ cells could stimulate pS2 expression, suggesting again that tamoxifen in the BT cells functions as an agonist. By western blot analysis, ER and ERBB2 levels in BT and BT/HerR1.0 cells were found to be similar (Fig. 1D). These results suggest that both the BT and BT/HerR cells exhibit wild-type ER function in response to $E_{2}$, but there is a clear difference in sensitivity to tamoxifen in both growth and ER functional assays.

\section{Attenuated cross-phosphorylation between ER and Her2 downstream components in BT and BT/HerR cells}

The crosstalk between ER and ERBB2 involves ER nongenomic activity and is thought to be very important in the development of tamoxifen resistance (Pietras et al. 1995, Shou et al. 2004). To explore the mechanism of differential sensitivities to tamoxifen in the BT and $\mathrm{BT} / \mathrm{HerR}$ cells, the nongenomic activity of ER in BT and BT/HerR1.0 cells was examined. Our previous studies have found that the total and phosphorylated ERBB2 levels in BT/HerR cells are not significantly changed compared with BT cells, and signaling through the PI3K/Akt pathway is sustained in the presence of Herceptin in BT/HerR (Chan et al. 2005). To further explore our previous findings, we examined the changes of downstream components of the ERBB2 pathway, such as MAPK and Akt, in the presence of $\mathrm{E}_{2}$ and tamoxifen. As shown by western blot analysis (Fig. 2A and B), after a 20-min treatment with $\mathrm{E}_{2}$ and tamoxifen, both $\mathrm{E}_{2}$ and tamoxifen could stimulate phosphorylation of MAPK and
Akt in BT cells compared with the DMSO control. However, while the basal phosphorylated Akt was slightly elevated in BT/HerR1.0 compared with BT cells (Fig. 2B), both $\mathrm{E}_{2}$ and tamoxifen in these cells could not stimulate phosphorylation of MAPK and Akt. These results indicate that ER nongenomic activity in $\mathrm{BT} / \mathrm{HerR}$ cells is attenuated, and tamoxifen could function as an agonist in BT, but not in BT/HerR cells. To determine the effect of attenuated ER nongenomic activity on ER function in BT/HerR cells, ER phosphorylation in BT and BT/HerR1.0 cells treated with or without $\mathrm{E}_{2}$ or tamoxifen was examined using a specific anti-phosphoSer118 or Ser167 ESR1 antibody. In BT cells, activated MAPK by $\mathrm{E}_{2}$ and tamoxifen could lead to potentiation of phospho-ESR1 at Ser118, which was previously reported to be phosphorylated by MAPK (Kato et al. 1995). However, $\mathrm{E}_{2}$ and tamoxifen-stimulated phospho-ESR1 at Ser118 was found to be attenuated in the BT/HerR1.0 cells compared with that in BT cells. Under our experimental conditions, phosphorylation of ESR1 at Ser167, which could be phosphorylated by Akt (Campbell et al. 2001), could not be detected in either cell line (data not shown). Our results are similar to those of a previous study on BT cells (Wang et al. 2005), implying that Ser-167 phosphorylation is not abundant in the BTand BT/HerR cells. The effects of $\mathrm{E}_{2}$ and tamoxifen treatments on phosphorylated levels of MAPK, Akt, and ER were still significant after $24 \mathrm{~h}$ (Fig. 2C and D), indicating that they are not transient. In addition, $\mathrm{E}_{2}$ treatment for $24 \mathrm{~h}$ could down-regulate the total ER level and tamoxifen treatment for $24 \mathrm{~h}$ could up-regulate the total ER level in these two cell lines (Fig. 2C and D), which is consistent with other's findings (Wang et al. 2005).

\section{Localization of ER in BT and BT/HerR cells}

Since subcellular localization of ER has been linked to the responsiveness of breast cancer cells to tamoxifen (Yang et al. 2004), we analyzed the subcellular localization of ER in BT and BT/HerR1.0 cells by immunofluorescence and confocal studies. In regular media, more ER in BT cells was found to localize in the membrane/cytoplasm than in the nucleus (Fig. 3A), but in BT/HerR1.0 cells, more ER localized in the nucleus (Fig. 3B), indicating that the genomic pathway of ER in BT/HerR cells is more important than the nongenomic pathway. To further confirm these results, cells were deprived of endogenous $\mathrm{E}_{2}$ for 3 days and then treated with $1 \mu \mathrm{M} \mathrm{E}_{2}$ for $1 \mathrm{~h}$, and the effects of $\mathrm{E}_{2}$ on ER localization were examined. Estrogen stimulation could promote ER translocation from the nucleus to the membrane/cytoplasm in BT cells (Fig. 3A), but from the membrane/cytoplasm to the nucleus in BT/HerR1.0 cells (Fig. 3B). This different localization of ER in BT and BT/HerR1.0 cells can also be observed using another ER antibody (ER [6F11] 

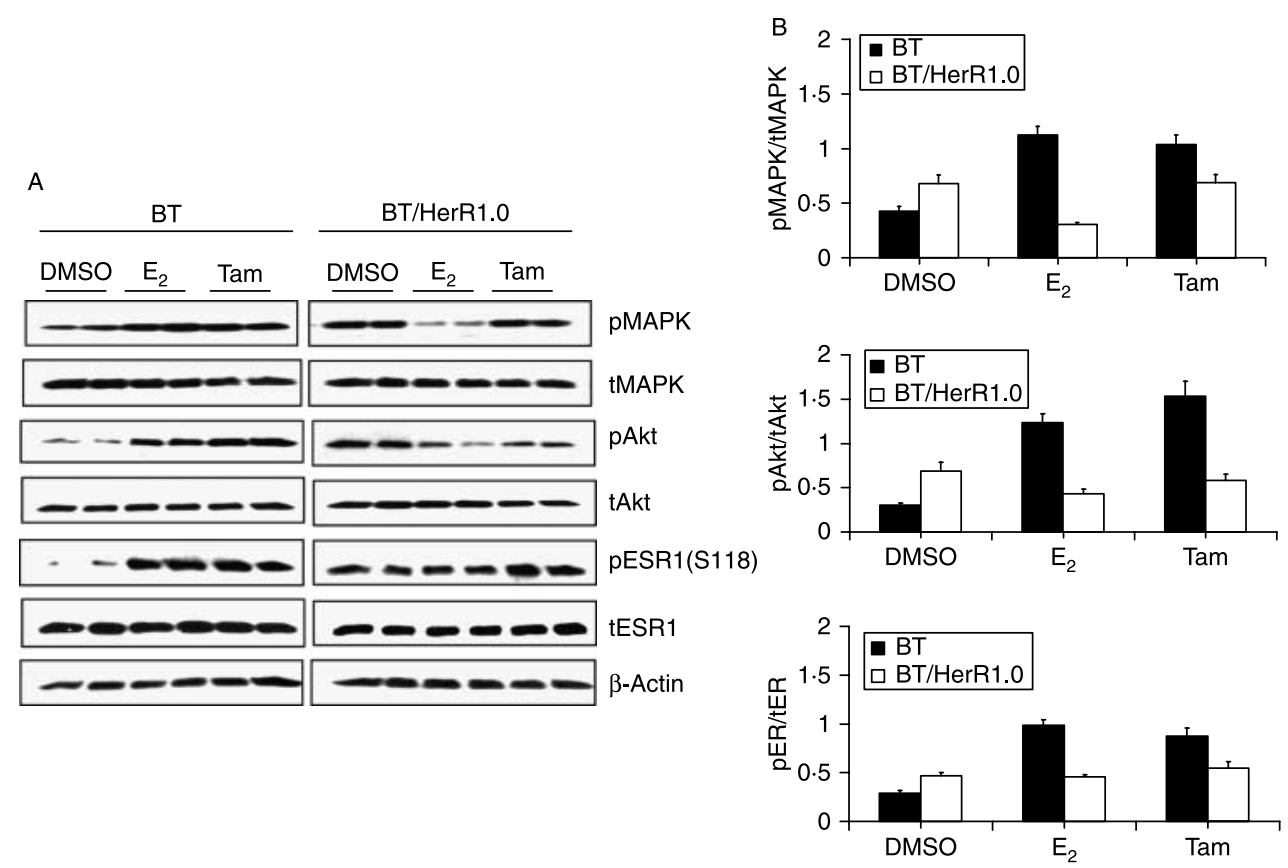

D

C
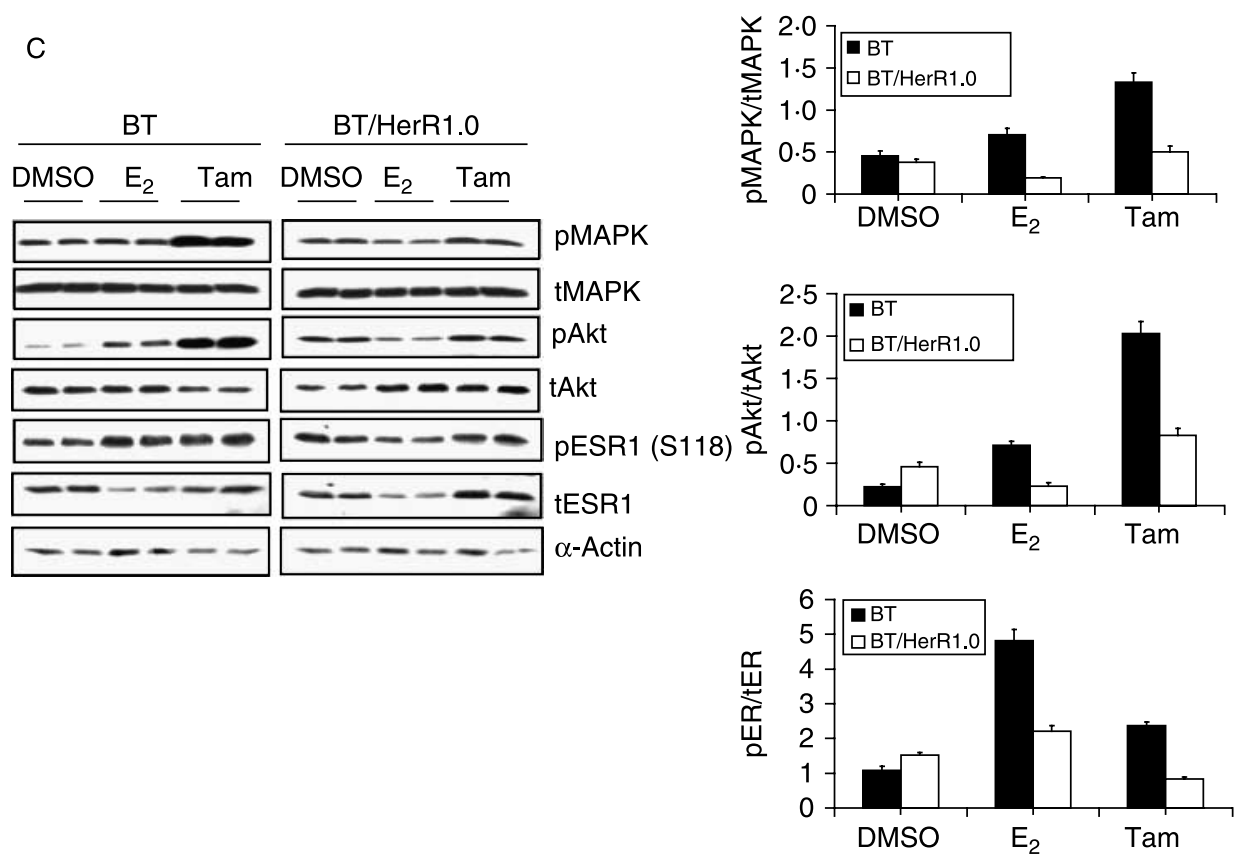

Figure 2 Cross-phosphorylation between ER and ERBB2 downstream components in the BT and $\mathrm{BT} / \mathrm{HerR}$ cells. BT and BT/HerR1.0 cells were deprived of steroid for $72 \mathrm{~h}$ and serum starved for $24 \mathrm{~h}$, and then treated with DMSO, $1 \mathrm{nM} \mathrm{E}_{2}, 1 \mu \mathrm{M}$ tamoxifen (Tam) for $20 \mathrm{~min}(\mathrm{~A}$ and $\mathrm{B}$ ) or $24 \mathrm{~h}$ (C and D). Levels of total $(t)$ and phosphorylated $(p)$ MAPK, Akt and ESR1 at Ser118 were measured in cellular extracts by immunoblot analysis. $\beta$-actin was used as a control for equal loading and transfer. Figures are representative of three experiments. Phosphorylation levels of the experiments were estimated by densitometry, and were calculated as the ratio of pMAPK/tMAPK, pAkt/tAkt, or pER/tER. 
A

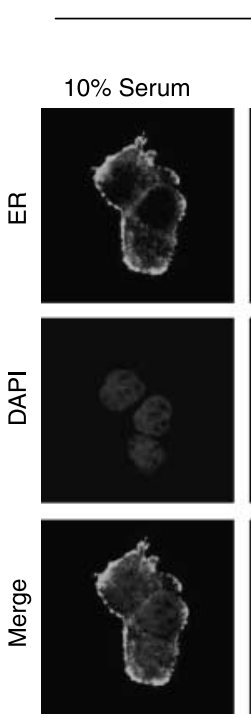

BT

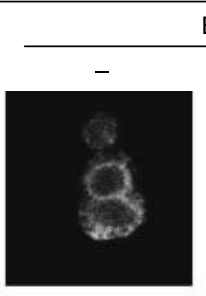

$\mathrm{E}_{2}$
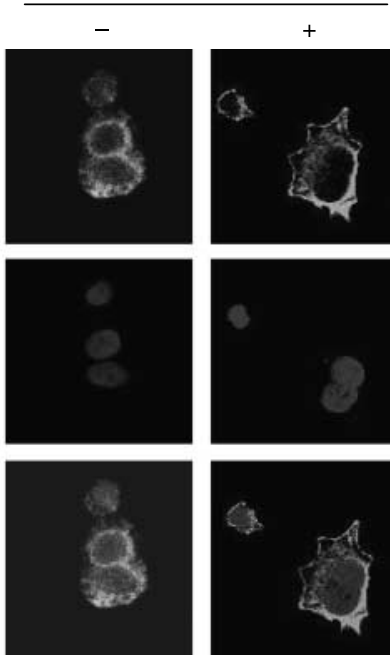
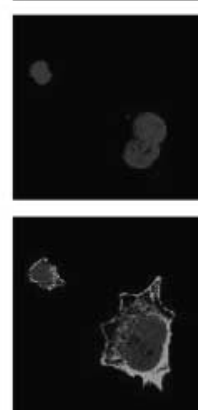

B

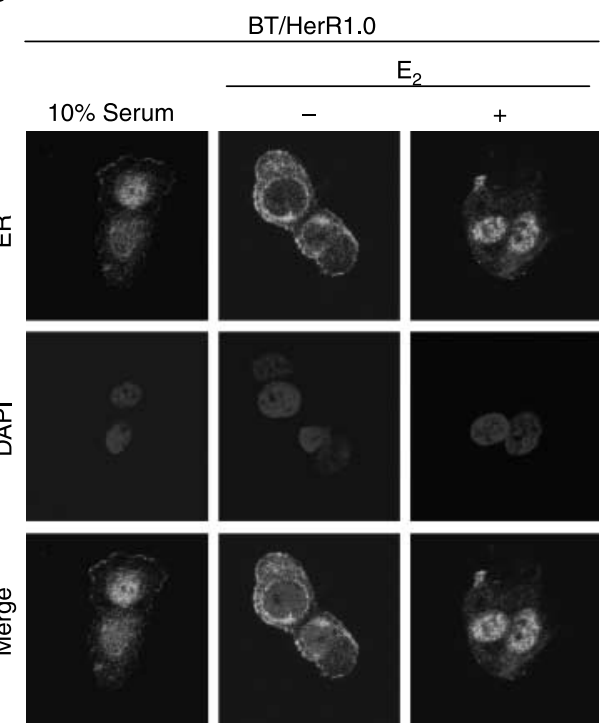

Figure 3 Subcellular localization of ER in BT (A) and BT/HerR cells (B). BT and BT/HerR1.0 cells were cultured in regular media (10\% serum) or steroid-deprived media in the absence $(-)$ or presence $(+)$ of $1 \mathrm{nM} \mathrm{E}_{2}$. Cellular localization of ER was determined by indirect immunofluorescence as indicated in the 'Materials and methods'. Confocal microscopy was used to show the presence of ER in the cytoplasmic compartment and nucleus. The cells were deprived of steroid for $72 \mathrm{~h}$ and then stimulated with $\mathrm{E}_{2}$ for $1 \mathrm{~h}$ to examine the effects on ER localization. The (ER) cells labeled with ER (F-10) antibody; (DAPI) nucleus counterstained with DAPI; (merge) ER localization assessed by spectral overlap of the images in top and middle panels.

antibody, Abcam) for the same analysis (Supplementary Fig. 1, see Supplementary data in the online version of the Journal of Molecular Endocrinology at http://jme. endocrinology-journals.org/content/vol41/issue/5). These results show that ER subcellular localization in $\mathrm{BT} /$ HerR cells is different from BT cells, which suggests that enhancement of the ER genomic pathway is one of the potential mechanisms related with increased sensitivity to tamoxifen in the BT/HerR cells.

\section{Different recruitment of nuclear receptor coactivator or corepressor on pS2 promoter by tamoxifen-bound ER in BT and BT/HerR cells}

One mechanism for tamoxifen resistance may be related to a change in the balance of association of ER with nuclear receptor coactivators or corepressors (Smith et al. 1997). Tamoxifen had been shown to function as an agonist in BT cells but an antagonist in BT/HerR cells (Figs $1 \mathrm{~A}$ and $\mathrm{C}$ and 3). Next, we employed ChIP assays to address if there was a different recruitment of nuclear receptor coactivator or corepressor on the pS2 promoter by tamoxifen-bound ER in these two kinds of cell lines. In BT cells, $\mathrm{E}_{2}$ induced occupancy of the pS2 promoter by ER and coactivator NCOA3. Tamoxifen, like $\mathrm{E}_{2}$, also induced occupancy by ER and coactivator NCOA3, but not corepressor NCOR1, which is consistent with its agonist function in these cells. In BT/HerR1.0 cells, $\mathrm{E}_{2}$ induced occupancy of the pS2 promoter by ER and coactivator NCOA3, but tamoxifen induced occupancy by ER and corepressor NCOR1 instead of coactivator NCOA3, which is consistent with the antagonist function of tamoxifen in these cells (Fig. 4). The different recruitment of nuclear receptor coactivator or corepressor on the pS2 promoter by tamoxifen-bound ER in BT and BT/HerR cells may explain the improvement of tamoxifen sensitivity in BT/HerR cells.

\section{Combination of tamoxifen and Herceptin improves the sensitivity of BT/HerR cells to Herceptin}

The crosstalk between ER and ERBB2 signaling pathways suggests that targeting both ER and growth factor receptors simultaneously could be a fruitful approach to the treatment of breast cancer. The combination of tamoxifen and Herceptin has been reported to synergistically inhibit the proliferation of BT cells (Argiris et al. 2004, Wang et al. 2005). Although BT/HerR cells are resistant to Herceptin, the sensitivity of these cells to tamoxifen was improved. Hence, we next investigated if the combination of tamoxifen and Herceptin could improve the sensitivity of BT/HerR cells to Herceptin. Compared with Herceptin or tamoxifen treatment alone, the combination of Herceptin with tamoxifen significantly inhibited the 


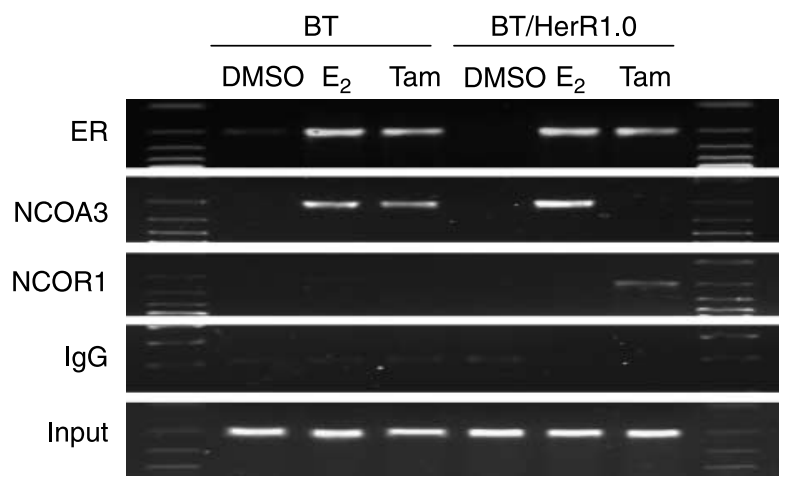

Figure 4 Different recruitment of nuclear receptor coactivator or corepressor on estrogen-responsive pS2 promoter by tamoxifenbound ER in the BT and BT/HerR cells. BT and BT/HerR1.0 cells were deprived of steroid for $72 \mathrm{~h}$ and serum starved for $24 \mathrm{~h}$, and then treated with DMSO, $1 \mathrm{nM} \mathrm{E}_{2}$, or $1 \mu \mathrm{M}$ tamoxifen (Tam) for $45 \mathrm{~min}$. Occupancy of the estrogen-responsive pS2 promoter by $\mathrm{ER}$, the coactivator NCOA3, and the corepressor NCOR1 was examined by ChIP assay as indicated in the 'Materials and methods'. Relevant pS2 promoter sequences were PCR amplified from complexes immunoprecipitated with each antibody. Input lane shows DNA that was PCR amplified from extracts before immunoprecipitation. Gels are representative of three experiments.

proliferation of the BT/HerR1.0 cells (Fig. 5A). Western blot analysis of signaling proteins showed that, consistent with other's findings (Wang et al. 2005), BT cells were very sensitive to Herceptin alone but not to tamoxifen alone and the combination of tamoxifen and Herceptin did not have any additional effect on phosphorylation of MAPK, Akt, or ESR1 at Ser118 (Fig. 5B). Though phosphorylation of MAPK, Akt, and
ESR1 at Ser118 was sustained in BT/HerR1.0 cells in the presence of Herceptin compared with BT cells, combination of Herceptin with tamoxifen significantly down-regulated phosphorylation of downstream signaling components of ERBB2 pathway and phospho- ESR1 at Ser118. These results indicate the combination of Herceptin with tamoxifen can sensitize BT/HerR cells to Herceptin, suggesting that the combined therapy with Herceptin and tamoxifen might be a viable therapeutic strategy for ER-positive and Herceptinresistant human breast cancer.

\section{Discussion}

Currently, Herceptin is the only ERBB2-targeted therapy approved by the US Food and Drug Administration (FDA) for the treatment of metastatic breast cancer. While Herceptin is a powerful drug, many patients who achieve an initial response to Herceptinbased regimens develop resistance within 1 year. In addition, the expensive prices of these drugs mean it is very important to explore new potential therapeutic strategies to improve the sensitivity of Herceptin. Besides the reported strategies of combination therapy for Herceptin resistance in breast cancer, Herceptinresistant cells have recently been shown to retain sensitivity to green tea polyphenol epigallocatechin-3gallate, providing a novel strategy for treatment of Herceptin-resistant breast cancers (Eddy et al. 2007). In the present study, we demonstrated the improvement
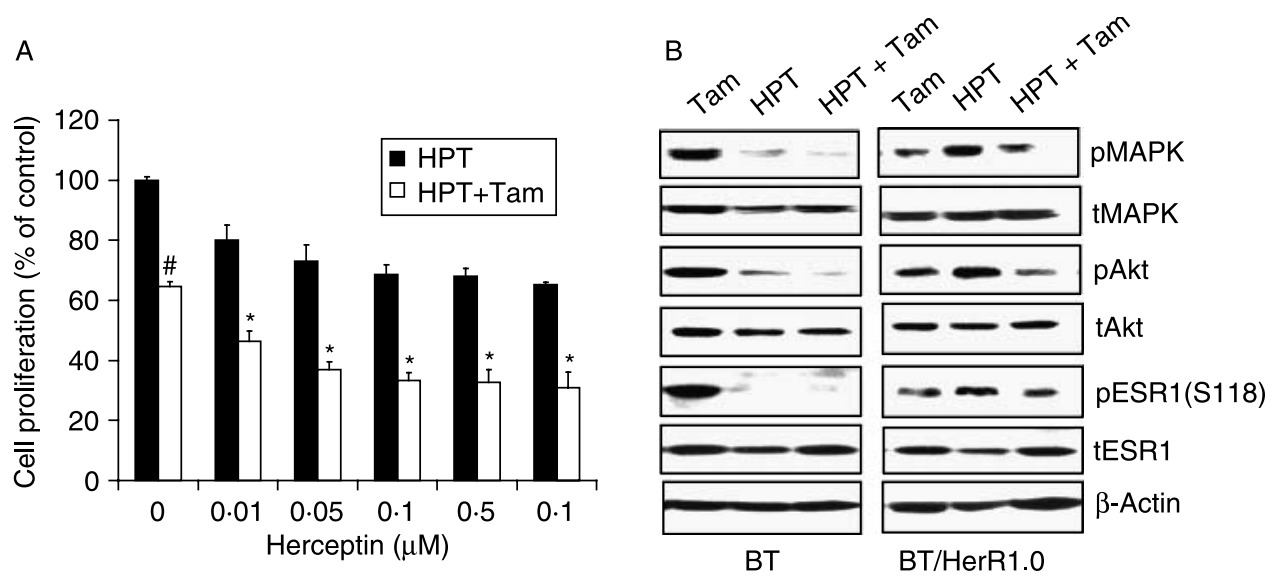

Figure 5 Combination of tamoxifen and Herceptin improves the sensitivity of BT/HerR cells to Herceptin. (A) BT and BT/HerR1.0 cells were deprived of steroid for $72 \mathrm{~h}$ and then incubated in the presence of $1 \mathrm{nM}$ $\mathrm{E}_{2}$ with increasing concentrations of Herceptin (HPT) or its combination with $1 \mu \mathrm{M}$ tamoxifen (Tam) for 6 days. Cell proliferation was expressed relative to optical density at $490 \mathrm{~nm}$ of cell line in the presence of $\mathrm{E}_{2 .}{ }^{\#} P<0.05$ compared with control in the absence of Herceptin and tamoxifen. ${ }^{\star} P<0.05$ combined agents versus Herceptin alone or tamoxifen alone. (B) BT and BT/HerR1.0 cells were treated with $1 \mu \mathrm{M}$ Herceptin or $1 \mu \mathrm{M}$ tamoxifen or both drugs (HPT + Tam) for $24 \mathrm{~h}$. Levels of total $(\mathrm{t})$ and phosphorylated $(\mathrm{p})$ MAPK, Akt, and ESR1 at Ser118 were measured in cellular extracts by western blot analysis. $\beta$-actin was used as a control for equal loading and transfer. Figures are representative of three experiments. 
of tamoxifen responses in BT/HerR cells and tried to explore the new therapeutic strategies to improve the sensitivity to Herceptin. Our results showed that there was a significant difference in response to tamoxifen between the BT and BT/HerR cells (Fig. 1A and B). $\mathrm{BT} /$ HerR cells were much more sensitive to tamoxifen than BT cells, and tamoxifen in these cells could function as an antagonist. By contrast, BT cells are resistant to tamoxifen, as observed by another laboratory (Zhou et al. 2007), and tamoxifen has a partial agonist function in these cells as well as in MCF-7/ ERBB2 cells, which have been reported to be resistant to tamoxifen (Kurokawa et al. 2000, Shou et al. 2004). In addition, we observed that the two kinds of cell lines had similar sensitivity to the pure ER antagonist, ICI. The different responses to tamoxifen and ICI in these cells are associated with the different mechanisms of the two agents affecting ER function. ICI may block both the transcriptional activating function 2 (AF-2) and transcriptional AF-1 of ER by attenuating dimerization of the receptor and accelerating receptor degradation, but tamoxifen only blocks AF2 of ER by competing with the receptor and allows activation of AF1 (Dowsett $e t$ al. 2005). Therefore, tamoxifen in the breast cancer cells only partially inactivates ER-regulated transcription or even has a partial agonist function in ERBB2-overexpressing cells such as MCF-7/ERBB2 (Kurokawa et al. 2000, Shou et al. 2004) and BT cells (Figs $1 \mathrm{~A}$ and C and 2). The different sensitivities to tamoxifen in the BT and $\mathrm{BT} /$ HerR cells are not attributable to differences in the ER and ERBB2 levels because total ER and ERBB2 levels in these two kinds of cells were similar (Fig. 1D). Considering the important role of the interaction of the ERBB2 and ER pathways in the development of tamoxifen resistance (Pietras et al. 1995, Shou et al. 2004), a change in crosstalk between ER- and ERBB2signaling pathways might be involved in this different response to tamoxifen in these two kinds of cell lines.

In ERBB2-overexpressing tumors, the crosstalk between the ER and ERBB2 pathways is associated with ER nongenomic activity, and the nongenomic rapid ER action in these cells may become more prominent. For example, tamoxifen has been shown to rapidly activate EGFR, ERBB2, Akt, and MAPK1/ MAPK in MCF-7/ERBB2 cells, establishing a cycle of cell survival and proliferation via bidirectional crosstalk between growth factor and ER signaling pathways (Shou et al. 2004). In BT cells, which express ER and overexpress ERBB2 and NCOA3 (Anzick et al. 1997, Yakes et al. 2002, Zhou et al. 2007), effects of $\mathrm{E}_{2}$ and tamoxifen on phosphorylation of downstream effectors of EGFR/ERBB2 are similar to those in MCF-7/ERBB2 cells (Shou et al. 2004; Fig. 2). In MCF-7/ERBB2 cells, the EGF receptor tyrosine kinase inhibitor gefitinib could eliminate this crosstalk and restore tamoxifen's antitumor effects, and Herceptin was also demonstrated to reverse tamoxifen resistance by reducing downstream MAPK/ESSRA/B signaling (Kurokawa et al. 2000). These findings suggest that Herceptin-mediated down-regulation of the ERBB2 pathway may be associated with increased sensitivity to tamoxifen. Our results showed the attenuated activation of Akt and MAPK by $\mathrm{E}_{2}$ or tamoxifen in BT/HerR1.0 cells (Fig. 2). Consistent with the attenuated change in phosphorylated MAPK, $\mathrm{E}_{2}$, and tamoxifen-stimulated phosphoESR1 at Ser118 was also attenuated. These results suggest that the nongenomic activities of ER in $\mathrm{BT} /$ HerR cells is decreased, which leads to the attenuation of the crosstalk between growth factor and ER-signaling pathways, and may be involved in the increased sensitivity to tamoxifen in the BT/HerR cells. The reason for the attenuated activation of Akt and MAPK by $\mathrm{E}_{2}$ or tamoxifen in BT/HerR cells is currently not clear and may be associated with chronic Herceptin exposure. For example, phosphorylated Akt levels in the presence of Herceptin in BT/HerR cells has been found to be lower than in BT cells in the absence of Herceptin (Chan et al. 2005). However, in the absence of Herceptin, the basal phosphorylated Akt level in BT/HerR1.0 cells was not decreased and inversely was somehow increased, compared with BT cells (Fig. 2). Hence, the precise mechanism of attenuated activation of Akt and MAPK by $\mathrm{E}_{2}$ or tamoxifen in the BT/HerR cells will require further research.

Besides the crosstalk between ER and ERBB2, recent research has shown that subcellular localization of ER may play a mechanistic role in determining the responsiveness of breast cancer cells to tamoxifen (Yang et al. 2004). ERBB2 overexpression in MCF-7/ ERBB2 cells promotes the relocalization of ER from the nucleus to the cytoplasm, enhances interactions of ER with ERBB2, inhibits ER transactivation function, and induces resistance to tamoxifen-mediated growth inhibition of breast cancer cells. Conversely, downregulation of ERBB2 by Herceptin leads to suppression of MAPK stimulation, restoration of ER to the nucleus, and potentiation of the growth-inhibitory action of tamoxifen. Different subcellular localization of ER in specific cells represents the different activating status of genomic pathway or nongenomic pathway of ER. Our data showed different subcellular localization of ER in the BT and BT/HerR1.0 cells, which suggest that exclusion of ER from the nucleus in BT cells might act to deprive the antiestrogenic agents of their target in the proper cellular compartment. On the other hand, entering into the nucleus of ER from the cytoplasm in BT/HerR1.0 cells might indicate a restoration of the ER genomic pathway, leading to a subsequent increased sensitivity to tamoxifen (Figs 1 and 3). The restoration of the ER genomic pathway in BT/HerR cells is not due to the changes in ERBB2 and ER levels because the steady-state ER, total and phosphorylated ERBB2 levels 
in the BT and BT/HerR1.0 cells are the same (Fig. 1D). On the other hand, our results suggest that attenuated activation of Akt and MAPK by $\mathrm{E}_{2}$ or tamoxifen could confer the suppressed cross-phosphorylation between ER and ERBB2 downstream components, which may be associated with the restoration of the ER genomic pathway and subsequent improvement of tamoxifen sensitivity in $\mathrm{BT} /$ HerR cells.

It is well known that ER directly binds to estrogen response element residing in the promoter region of the target gene and, by recruiting co-regulatory proteins, regulates gene transcription. In addition to the activation of ER phosphorylation, activated MAPK in tamoxifen-resistant cells can phosphorylate ER coactivator NCOA3 (Shou et al. 2004) and the resistant status may be related to a change in the balance of the association of ER with coactivators or corepressors (Smith et al. 1997). A recent report describes how the forced overexpression of ERBB2 in $\mathrm{E}_{2}$-dependent, tamoxifen-sensitive MCF-7 cells results in tamoxifen resistance (Shou et al. 2004). In MCF-7 cells, tamoxifen induces occupancy of the pS2 promoter by ER, corepressor NCOR1, and HDAC3. However, in MCF7/ERBB2 cells, tamoxifen induces occupancy by ER and coactivator complexes including NCOA3, P300, and CBP, resulting in the formation of acetylated histones, thereby explaining agonist effects of tamoxifen on steroid-regulated gene expression. Given the improvement of the ER genomic pathway and increased tamoxifen sensitivity in BT/HerR cells, recruitment of nuclear receptor coactivator or corepressor on pS2 promoter by tamoxifen-bound ER in these two kinds of cell lines should be different. Consistent with the observation in MCF-7 and MCF-7/ERBB2 cells, our results showed that tamoxifen in the BT cells induced occupancy by ER and coactivator NCOA3, which corresponds to its agonist function in these cells, whereas tamoxifen in the BT/HerR1.0 cells induced occupancy by corepressor NCOR1. The replacement of coactivator complexes with corepressor complexes in the presence of tamoxifen-bound ER is consistent with an antagonist function of tamoxifen in the BT/HerR1.0 cells. The different recruitment of nuclear receptor coactivator or corepressor on pS2 promoter in the BT and BT/HerR cells might explain the improvement of tamoxifen sensitivity in BT/HerR cells.

In summary, the results presented here show for the first time the enhancement of tamoxifen sensitivity in $\mathrm{BT} /$ HerR cells, relative to BT cells, and the following mechanisms are implicated: a) attenuated activation of MAPK and Akt pathway by $\mathrm{E}_{2}$ and tamoxifen, indicating the decreased ER nongenomic activity; b) different subcelluar localization from BT cells, indicating improvement of genomic pathway of ER; and c) the replacement of coactivator complexes with corepressor complexes on ERE-containing promoters in the presence of tamoxifen-bound ER. These results serve as direct evidence for the antagonist activity of tamoxifen in the BT/HerR cells. Although there is an inverse relationship between the degree of ER positivity and ERBB2 expression, a significant proportion of human breast cancers are both ER-positive and overexpressing ERBB2 (Berry et al. 2000). Compared with ER-positive, ERBB2-overexpressing, and tamoxifen-resistant BT cells, the increased sensitivity to classical endocrine therapy such as tamoxifen in the Herceptin-resistant BT/HerR cells suggests that ER genomic pathway in these cells can be improved, thus allowing tamoxifen therapy to be effective again. In spite of the increased sensitivity to tamoxifen in the BT/HerR cells, our data imply that monotherapy with tamoxifen might have only modest benefits on BT/HerR cells, i.e., $60 \%$ inhibition of proliferation in BT/HerR1.0 cells compared with the control (Figs $1 \mathrm{~B}$ and 5A). However, combination of tamoxifen and Herceptin was demonstrated to increase the sensitivity of BT/HerR1.0 cells to Herceptin (Fig. 5), suggesting that combined therapy with Herceptin and tamoxifen might be a viable therapeutic strategy for Herceptin-resistant human breast cancer cells.

\section{Declaration of interest}

The authors declare that there is no conflict of interest that could be perceived as prejudicing the impartiality of the research reported.

\section{Funding}

The research project described in this paper has been supported by NIH grants ES08258 (SC), CA44735 (SC), and CA33572 (the COH Cancer Center grant).

\section{References}

Anzick SL, Kononen J, Walker RL, Azorsa DO, Tanner MM, Guan XY, Sauter G, Kallioniemi OP, Trent JM \& Meltzer PS 1997 AIB1, a steroid receptor coactivator amplified in breast and ovarian cancer. Science 277 965-968.

Argiris A, Wang CX, Whalen SG \& DiGiovanna MP 2004 Synergistic interactions between tamoxifen and trastuzumab (Herceptin). Clinical Cancer Research 10 1409-1420.

Berry DA, Muss HB, Thor AD, Dressler L, Liu ET, Broadwater G, Budman DR, Henderson IC, Barcos M, Hayes D et al. 2000 HER$2 /$ neu and p53 expression versus tamoxifen resistance in estrogen receptor-positive, node-positive breast cancer. Journal of Clinical Oncology 18 3471-3479.

Bradford MM 1976 A rapid and sensitive method for the quantitation of microgram quantities of protein utilizing the principle of protein-dye binding. Analytical Biochemistry 72 248-254.

Campbell RA, Bhat-Nakshatri P, Patel NM, Constantinidou D, Ali S \& Nakshatri H 2001 Phosphatidylinositol 3-kinase/AKT-mediated activation of estrogen receptor alpha: a new model for anti-estrogen resistance. Journal of Biological Chemistry 276 9817-9824. 
Carter P, Presta L, Gorman CM, Ridgway JB, Henner D, Wong WL, Rowland AM, Kotts C, Carver ME \& Shepard HM 1992 Humanization of an anti-p185HER2 antibody for human cancer therapy. PNAS 89 4285-4289.

Chan CT, Metz MZ \& Kane SE 2005 Differential sensitivities of trastuzumab (Herceptin)-resistant human breast cancer cells to phosphoinositide-3 kinase (PI-3K) and epidermal growth factor receptor (EGFR) kinase inhibitors. Breast Cancer Research and Treatment 91 187-201.

Clark AS, West K, Streicher S \& Dennis PA 2002 Constitutive and inducible Akt activity promotes resistance to chemotherapy, trastuzumab, or tamoxifen in breast cancer cells. Molecular Cancer Therapeutics 1 707-717.

Dieras V, Beuzeboc P, Laurence V, Pierga JY \& Pouillart P 2001 Interaction between Herceptin and taxanes. Oncology 61 (Suppl 2) 43-49.

Dowsett M, Nicholson RI \& Pietras RJ 2005 Biological characteristics of the pure antiestrogen fulvestrant: overcoming endocrine resistance. Breast Cancer Research and Treatment 93 (Suppl 1) S11-S18.

Eddy SF, Kane SE \& Sonenshein GE 2007 Trastuzumab-resistant HER2driven breast cancer cells are sensitive to epigallocatechin-3 gallate. Cancer Research 67 9018-9023.

Kato S, Endoh H, Masuhiro Y, Kitamoto T, Uchiyama S, Sasaki H, Masushige S, Gotoh Y, Nishida E, Kawashima H et al. 1995 Activation of the estrogen receptor through phosphorylation by mitogenactivated protein kinase. Science 270 1491-1494.

Kurokawa H, Lenferink AE, Simpson JF, Pisacane PI, Sliwkowski MX, Forbes JT \& Arteaga CL 2000 Inhibition of HER2/neu (erbB-2) and mitogen-activated protein kinases enhances tamoxifen action against HER2-overexpressing, tamoxifen-resistant breast cancer cells. Cancer Research 60 5887-5894.

Lenferink AE, Simpson JF, Shawver LK, Coffey RJ, Forbes JT \& Arteaga CL 2000 Blockade of the epidermal growth factor receptor tyrosine kinase suppresses tumorigenesis in MMTV/Neu + MMTV/TGF- $\alpha$ bigenic mice. PNAS 97 9609-9614.

Lou G, Li Y, Chen B, Chen M, Chen J, Liao R, Zhang Y, Wang Y \& Zhou D 2007 Functional analysis on the 5 '-flanking region of human FXR gene in HepG2 cells. Gene 396 358-368.

Lu Y, Zi X, Zhao Y, Mascarenhas D \& Pollak M 2001 Insulin-like growth factor-I receptor signaling and resistance to trastuzumab (Herceptin). Journal of the National Cancer Institute 93 1852-1857.

Moasser MM, Basso A, Averbuch SD \& Rosen N 2001 The tyrosine kinase inhibitor ZD1839 ('Iressa') inhibits HER2-driven signaling and suppresses the growth of HER2-overexpressing tumor cells. Cancer Research 61 7184-7188.

Nahta R, Yu D, Hung MC, Hortobagyi GN \& Esteva FJ 2006 Mechanisms of disease: understanding resistance to HER2-targeted therapy in human breast cancer. Nature Clinical Practice and Oncology 3 269-280.

Pietras RJ, Arboleda J, Reese DM, Wongvipat N, Pegram MD, Ramos L, Gorman CM, Parker MG, Sliwkowski MX \& Slamon DJ 1995 HER-2 tyrosine kinase pathway targets estrogen receptor and promotes hormone-independent growth in human breast cancer cells. Oncogene 10 2435-2446.

Price-Schiavi SA, Jepson S, Li P, Arango M, Rudland PS, Yee L \& Carraway KL 2002 Rat Muc4 (sialomucin complex) reduces binding of anti-ErbB2 antibodies to tumor cell surfaces, a potential mechanism for herceptin resistance. International Journal of Cancer 99 783-791.

Schiff R, Massarweh SA, Shou J, Bharwani L, Mohsin SK \& Osborne CK 2004 Cross-talk between estrogen receptor and growth factor pathways as a molecular target for overcoming endocrine resistance. Clinical Cancer Research 10 331S-336S.
Shou J, Massarweh S, Osborne CK, Wakeling AE, Ali S, Weiss H \& Schiff R 2004 Mechanisms of tamoxifen resistance: increased estrogen receptor-HER2/neu cross-talk in ER/HER2-positive breast cancer. Journal of the National Cancer Institute 96 926-935.

Slamon DJ, Godolphin W, Jones LA, Holt JA, Wong SG, Keith DE, Levin WJ, Stuart SG, Udove J, Ullrich A et al. 1989 Studies of the HER-2/neu proto-oncogene in human breast and ovarian cancer. Science 244 707-712.

Smith CL, Nawaz Z \& O'Malley BW 1997 Coactivator and corepressor regulation of the agonist/antagonist activity of the mixed antiestrogen, 4-hydroxytamoxifen. Molecular Endocrinology 11 $657-666$.

Song RX, McPherson RA, Adam L, Bao Y, Shupnik M, Kumar R \& Santen RJ 2002 Linkage of rapid estrogen action to MAPK activation by ERalpha-Shc association and Shc pathway activation. Molecular Endocrinology 16 116-127.

Sun M, Paciga JE, Feldman RI, Yuan Z, Coppola D, Lu YY, Shelley SA, Nicosia SV \& Cheng JQ 2001 Phosphatidylinositol-3-OH kinase (PI3K)/AKT2, activated in breast cancer, regulates and is induced by estrogen receptor alpha $(\mathrm{ER} \alpha)$ via interaction between $\mathrm{ER} \alpha$ and PI3K. Cancer Research 61 5985-5991.

Tang CK, Perez C, Grunt T, Waibel C, Cho C \& Lupu R 1996 Involvement of heregulin-beta2 in the acquisition of the hormoneindependent phenotype of breast cancer cells. Cancer Research $\mathbf{5 6}$ $3350-3358$

Tseng PH, Wang YC, Weng SC, Weng JR, Chen CS, Brueggemeier RW, Shapiro CL, Chen CY, Dunn SE, Pollak M et al. 2006 Overcoming trastuzumab resistance in HER2-overexpressing breast cancer cells by using a novel celecoxib-derived phosphoinositide-dependent kinase-1 inhibitor. Molecular Pharmacology 70 1534-1541.

Vogel CL, Cobleigh MA, Tripathy D, Gutheil JC, Harris LN, Fehrenbacher L, Slamon DJ, Murphy M, Novotny WF, Burchmore M et al. 2002 Efficacy and safety of trastuzumab as a single agent in first-line treatment of HER2-overexpressing metastatic breast cancer. Journal of Clinical Oncology 20 719-726.

Wang CX, Koay DC, Edwards A, Lu Z, Mor G, Ocal IT \& Digiovanna MP 2005 In vitro and in vivo effects of combination of trastuzumab (Herceptin) and Tamoxifen in breast cancer. Breast Cancer Research and Treatment 92 251-263.

Wong CW, McNally C, Nickbarg E, Komm BS \& Cheskis BJ 2002 Estrogen receptor-interacting protein that modulates its nongenomic activity-crosstalk with Src/Erk phosphorylation cascade. PNAS 99 14783-14788.

Yakes FM, Chinratanalab W, Ritter CA, King W, Seelig S \& Arteaga CL 2002 Herceptin-induced inhibition of phosphatidylinositol-3 kinase and Akt Is required for antibody-mediated effects on p27, cyclin D1, and antitumor action. Cancer Research 62 4132-4141.

Yang Z, Barnes CJ \& Kumar R 2004 Human epidermal growth factor receptor 2 status modulates subcellular localization of and interaction with estrogen receptor alpha in breast cancer cells. Clinical Cancer Research 10 3621-3628.

Zhou Y, Yau C, Gray JW, Chew K, Dairkee SH, Moore DH, Eppenberger U, Eppenberger-Castori S \& Benz CC 2007 Enhanced NFKB and AP-1 transcriptional activity associated with antiestrogen resistant breast cancer. BMC Cancer 759 .

Received in final form 20 August 2008

Accepted 3 September 2008

Made available online as an Accepted Preprint 3 September 2008 